\title{
Time Reversal of Electromagnetic Waves and Telecommunication
}

\author{
G. Lerosey, ${ }^{1}$ J. de Rosny, ${ }^{1}$ A. Tourin,${ }^{1}$ A. Derode,${ }^{1}$ G. Montaldo, ${ }^{1}$ and M. Fink ${ }^{1}$
}

Time reversal (TR) communication in various configurations (SISO, MISO or MIMO) is studied. Especially, we report an experimental demonstration of time-reversal focusing with electromagnetic waves in a SISO scheme. An antenna transmits a $1 \mu \mathrm{s}$ electromagnetic pulse at a central frequency of $2.45 \mathrm{GHz}$ in a high-Q cavity. Another antenna records the strongly reverberated signal. The time-reversed wave is built and transmitted back by the same antenna acting now as a time-reversal mirror. The wave is found to converge to its initial source and is compressed in time. The quality of focusing is determined by the frequency bandwidth and the spectral correlations of the field within the cavity. A spatial focusing of the compressed pulse is also shown. This experiment is the first step for a communication scheme based on time-reversal. It would be very interesting for UWB communication in complex media since TR would permit to compensate for delay spreading. MISO and MIMO TR communications are discussed on the basis of small-scale experiments with ultrasound. Especially, the binary error rate of the method is studied as a function of both data rate and external noise. A simple theoretical approach explains the results.

\section{Introduction}

Nowadays, telecommunication systems demand higher and higher data transfer rates. In the recent past, intelligent coding [Proakis, 2000] led to the increase of the transfer rate. Yet a further increase will require larger bandwidths [Paulraj et al, 2004]. A major problem is that, as the bandwidth is enlarged, electro-magnetic waves undergo diffraction, reverberation and scattering, which results in fading and delay spreading. An initially short pulse that propagates in a complex environment spread in time on a typical duration $\tau$ which can be much larger than the inverse of the bandwidth. Usually the time spreading is due to one or several wave reflections off obstacles inside buildings or in the streets. This phenomenon induces Inter Symbol Interference (ISI) when the distance between symbols is shorter than the spreading time. ISI is an important problem to overcome, particularly in the field of UWB commu-

\footnotetext{
${ }^{1}$ Laboratoire Ondes et Acoustique, ESPCI, Université Paris VII, UMR 7587, 10 rue Vauquelin, 75005 Paris, France.
}

Copyright 2006 by the American Geophysical Union. 0048-6604/06/\$11.00 nications where the symbol length is potentially very short.

A few years ago, an original technique based on time reversal [Fink, 1997] of acoustic waves has been proposed for acoustic communications in shallow water reverberating environments [Edelmann et al, 2002]. Later the TR communication technique was generalized to MIMO schemes for ultrasonic (sub-millimeter wavelength) multi-user communication through a strongly multiple scattering medium [Derode et al, 2003]. In these experiments, the propagation medium consisted in a 40-cm large and 35-mm thick forest made of thousands of parallel steel rods. In such a complex medium, the "iterative time reversal" [Montaldo et al, 2004], an iterative algorithm that robustly converges to the inverse filter, was also successfully tested.

More recently, the first time-reversal experiment was reported for electromagnetic waves in the 2.45 $\mathrm{GHz}$ band [Lerosey et al, 2004]. This suggests that the techniques developed for ultrasound might be used for the electromagnetic case. It could be an interesting challenge because in many real environments (buildings or cities), microwaves with wavelengths between 10 and $30 \mathrm{~cm}$ are scattered off objects such as walls, desks, cars and so on, which produces a multitude of paths from the transmitter to the receiver. In such situations, a time re- 
versal antenna should be able not only to compensate for these multipaths, but also to increase the information transfer rate thanks to the many reflections/reverberations. Indeed, it has been shown that reflections could be taken advantage of: in the case of multiple antenna arrays, reflections yield more independent propagation channels and therefore help to increase the capacity [Moustakas et al, 2000]. A basic experimental proof of such a principle, using phase conjugation, can be found in [Henty and Stancil, 2004].

From a theoretical point of view, time-reversal of electromagnetic waves has been discussed in ref. [ $\mathrm{Ch}$ eney et al, 2001] and in ref. [Oestges et al, 2005]. The potential interest of time reversal in real environments has been confirmed by two recent papers. Using broadband radio wireless measurements in an office space with many cubicles, Emami et al.[Emami et al, 2005] have computed the time-reversed field around a given receiver when a single transmitter is used in a time-reversal mode. They retrieved the remarkable space-time focusing properties of timereversal. [Nguyen et al, 2005] as well as [Kyritsi et $a l, 2004]$ have studied the feasibility of applying TR techniques in multiple antenna wireless communication systems based on outdoor measurements. They showed that TR in wireless communication mitigates the effect of channel dispersion and reduce the cochannel interference.

In this paper, we discuss time-reversal communication in various configurations: SISO, MISO or MIMO. In the next section, we recall the principles of time reversal and its interest for communication purposes. The third section reports an experiment in which wideband electromagnetic waves are time reversed. It has been performed around $2.45 \mathrm{GHz}$ in a reverberant chamber with a one-channel Time Reversal Mirror (TRM), which is the first step of a real SISO communication based on TR of electromagnetic waves. Section IV deals with MISO communication with ultrasound instead of electromagnetic waves. Indeed, as EM communication with multiple wide-band antennas is still difficult to implement, we studied MISO time reversal telecommunication in a one hundredth scale model of an indoor environment with MHz-ultrasonic waves. The Binary Error Rate (BER) of the method is studied as a function of both data rate and external noise. The communication channel is analyzed as Binary Symmetrical Communication Channels and TR theory in random media is applied to explain the results. Section V briefly discusses the extension of the method to MIMO communication.

\section{Basics of Time Reversal Communication}

In a propagation medium with reflecting surfaces, corners and so on, a wave can propagate between two points through multiple paths. In such a case, the response to a short pulse is spread in time because of the difference between travel times related to different paths (delay spread). One way to compensate for reverberations is to use Time Reversal. Basically the principle of the TR technique is the following: a transient wave $e(t)$ is emitted by a source located at $\mathbf{r}$. The wave propagates through a complex medium, which may be inhomogeneous, scattering or reverberating. The time dependence of the field is recorded by a set of $M$ antennas located at $\mathbf{a}_{\mathbf{i}}$ and digitized. The signals are time-reversed in a computer memory. Then each antenna sends back the reanalogized time-reversed version of the signal it had previously recorded. Due to reciprocity the wave back-propagates through the medium and finally refocuses at the initial source position (spatial focusing) and recovers its original duration (time compression).

Mathematically the TR communication process can be described in the following way: we assume that the propagation medium is reciprocal, i.e., $h\left(\mathbf{a}_{\mathbf{i}} \rightarrow \mathbf{r}, t\right)=h\left(\mathbf{r} \rightarrow \mathbf{a}_{\mathbf{i}}, t\right)$ where $h\left(\mathbf{r}_{\mathbf{1}} \rightarrow \mathbf{r}_{\mathbf{2}}, t\right)$ is the impulse response between $\mathbf{r}_{\mathbf{1}}$ and $\mathbf{r}_{\mathbf{2}}$. Hence if $e(t)$ is a Dirac pulse the signal at the user location is:

$$
\begin{array}{r}
\mathcal{R}_{T R}(t)= \\
\sum_{i=1}^{M} h\left(\mathbf{a}_{i} \rightarrow \mathbf{r},-t\right) \otimes h\left(\mathbf{a}_{i} \rightarrow \mathbf{r}, t\right)
\end{array}
$$

This signal consists of a peak of maximum amplitude at $t=0$ surrounded by sidelobes which in the following will be referred to as "noise". The more complex the propagation paths, the more $\mathcal{R}_{T R}$ is peaked around time $t=0$ [Derode et al, 2001; Blomgren et al, 2002; Draeger and Fink, 1997]. It has been shown that the peak-to-noise ratio (in amplitude) varies roughly as $\sqrt{M \Delta \nu / \delta \nu}$ where $\Delta \nu$ is the bandwidth of the initially emitted pulse and $\delta \nu$ defines the correlation frequency of the reverberated field, i.e. roughly the inverse of the spread time. The spread time being defined as the rms delay spread of the signal, $\tau_{r m s}$. 
The application of time reversal to telecommunications between a user located at point $\mathbf{r}$ and a base made of $M$ antennas can be described as follows: in a preliminary step, the user sends a short pulse and the base records the $M$ impulse responses between $\mathbf{r}$ and the antenna positions $\mathbf{a}_{i,(i=1 . . M)}$. Each impulse response is time-reversed and convolved with the message $s(t)$ before emission. For instance, in digital communication, the message $s(t)$ may consist of a random series of bits with a rate $1 / \delta t$. After time-reversal, the signal received by the user consists of a series of short pulses with the same rate, and little or no ISI, depending to the peak-to-noise ratio.

\section{Time Reversal of Electromagnetic Waves}

From a technical point of view, the main difficulty to transpose this technique to the electromagnetic case lies in the very high sampling frequencies that are needed to digitize radiofrequency (RF) signals. In this part, we propose a means to perform a time reversal experiment for electromagnetic waves using a sampling frequency much less than the carrier frequency. One way to perform time reversal for EM waves is to work with quasimonochromatic signals, i.e., to do a phase conjugation using the so-called three-wave or four-wave mixing in a non linear material in order to naturally produce the analogic phase-conjugated wave [Pepper, 1982; Chang et al, 1998]. Here, we want to perform a truly broadband time reversal for an electromagnetic pulse $m(t) \cos \left(2 \pi \nu_{0} t+\phi(t)\right)$, or equivalently $m_{I}(t) \cos \left(2 \pi \nu_{0} t\right)+m_{Q}(t) \sin \left(2 \pi \nu_{0} t\right)$. The carrier frequency is $\nu_{0}, m_{I}(t)$ and $m_{Q}(t)$ are the "baseband signals". The time-reversal operation can be directly performed on these baseband signals. The advantage is that the sampling frequency can be much lower than $2 \nu_{0}$.

\subsection{Experimental setup}

The experimental setup is the following (see Fig 1). We use two antenna working around $2.45 \mathrm{GHz}$ manufactured by Astron Inc. and two circuit boards (RD0310 from RFMD, effective bandwidth $5 \mathrm{MHz}$ ) implementing the physical layer of a WLAN (wireless local area network). On the transmit side, it enables us to transform a baseband signal coded on two analog inputs I and Q, respectively its in-phase component (cos) and its quadrature component ( $\sin$ ), to $R F$. On the receive side, it enables to go from the $\mathrm{RF}$ signal back to analog baseband I/Q.

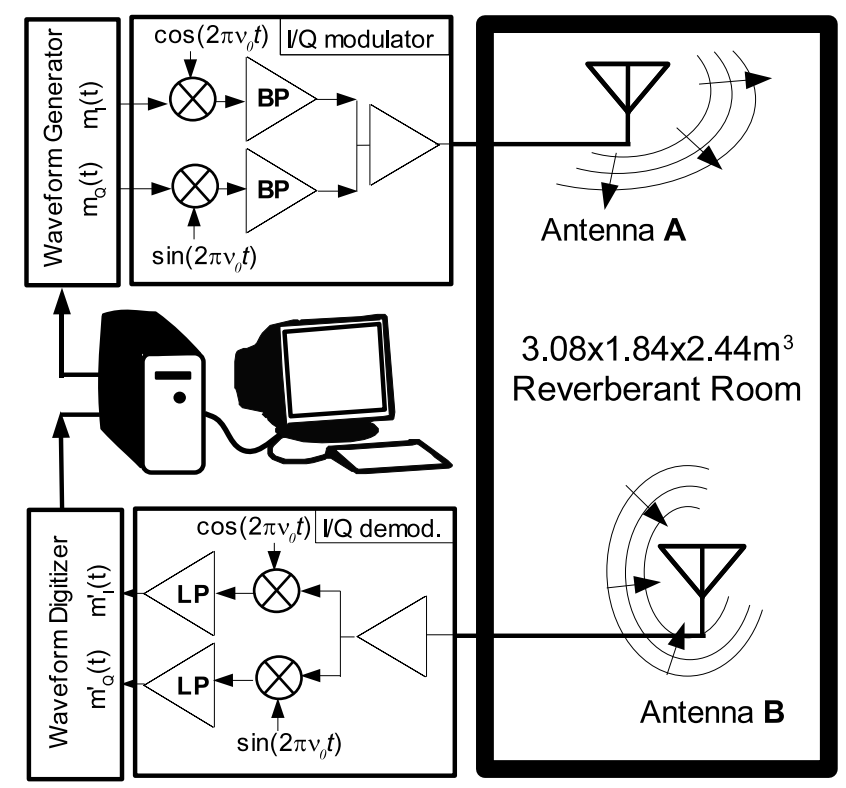

Figure 1. Sketch of a transmit/receive experiment. The baseband signal $m_{I}(t)$ (resp. $\left.m_{Q}(t)\right)$ is fed into a mixer that multiplies it by $\cos \left(2 \pi \nu_{0} t\right)$ (resp. $\left.\sin \left(2 \pi \nu_{0} t\right)\right)$, with $\nu_{0}=2.45 \mathrm{GHz}$. After amplification, the output RF signal is sent by antenna A, which is located in the reverberating room. The reverberated field is recorded by antenna B. Its spectrum is downshifted to the baseband by a multiplier and a low-pass filter. The resulting signals $m_{I}^{\prime}(t)$ and $m_{Q}^{\prime}(t)$ are sampled and stored in the computer memory. Then they can be time-reversed, re-analogized and, following the same principle, sent back by antenna $\mathrm{B}$ while antenna $\mathrm{A}$ acts now as a receiver.

The experiment takes place in a strongly reverberant cavity with dimensions $3.08 m \times 1.84 m \times 2.44 m$. Using an arbitrary waveform generator, we deliver a short pulse $m_{I}(t)$ (central frequency $3 \mathrm{MHz},-6 \mathrm{~dB}$ bandwidth $2 \mathrm{MHz}$ ) to the I analog input (cf. Fig. 2.(a)) of the transmit board. No signal is delivered to the $\mathrm{Q}$ analog input (Fig. 2.(a')). A mixer upconverts this signal to the $\mathrm{GHz}$ band and delivers $e(t)=m_{I}(t) \cos \left(2 \pi \nu_{0} t\right)$. Then the waveform $e(t)$ is transmitted by antenna A. After propagation, the signal $e^{\prime}(t)=m_{I}^{\prime}(t) \cos \left(2 \pi \nu_{0} t\right)+m_{Q}^{\prime}(t) \sin \left(2 \pi \nu_{0} t\right)$ is recorded by antenna $\mathrm{B}$ and downconverted to produce the I and $\mathrm{Q}$ components of the output signal $m_{I}^{\prime}(t)$ and $m_{Q}^{\prime}(t)$ that can be observed at the oscilloscope (Fig. 2. (b) and (b')). The received signals 
last more than $8 \mu s$, i.e. eight times longer than the initial baseband pulse, or 19600 periods of the RF wave. It means that the $\mathrm{RF}$ wave has traveled more than two $\mathrm{kms}$ in the $14-\mathrm{m}^{3}$ cavity, i.e. undergone a few thousands reflections.

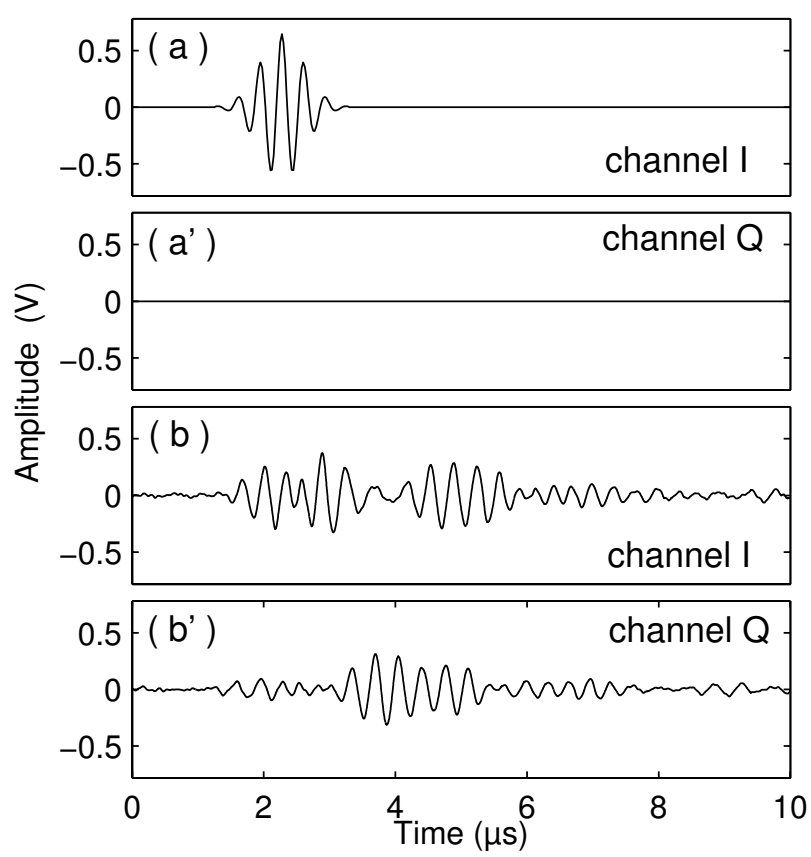

Figure 2. (a) and (a'): baseband representation (resp. $m_{I}(t)$ and $\left.m_{Q}(t)\right)$ of the signal transmitted by antenna A. (b) and (b'): baseband representation (i.e. $m_{I}^{\prime}(t)$ and $\left.m_{Q}^{\prime}(t)\right)$ of the signal reverberated inside the cavity and received by antenna $B$.

\subsection{Baseband Time Reversal}

Our goal is now to time-reverse the received radio signal $e^{\prime}(t)=m_{I}^{\prime}(t) \cos \left(2 \pi \nu_{0} t\right)+m_{Q}^{\prime}(t) \sin \left(2 \pi \nu_{0} t\right)$. Thus, the baseband I and Q signals $m_{I}^{\prime}$ and $m_{Q}^{\prime}$ are digitized by the oscilloscope at a $40-M H z$ sampling rate, sent to a computer and time-reversed. The wave carrier is conjugated too. The following step consists in re-analogizing the time-reversed I and Q signals, and encoding them on the phaseconjugated wave carrier: the resulting $\mathrm{RF}$ signal writes $m_{I}^{\prime}(-t) \cos \left(2 \pi \nu_{0} t\right)-m_{Q}^{\prime}(-t) \sin \left(2 \pi \nu_{0} t\right)=$ $e^{\prime}(-t)$. It is then transmitted back by antenna B. Af- ter propagation, the RF signal received at antenna $\mathrm{A}$ is downconverted to baseband.

\subsection{Temporal Compression}

As can be seen in Fig. 3.(a), the received signal on channel I is compressed in time and recovers its initial duration. Note that the acquisition window has been arbitrarily chosen in order to center the focused pulse within the figure.

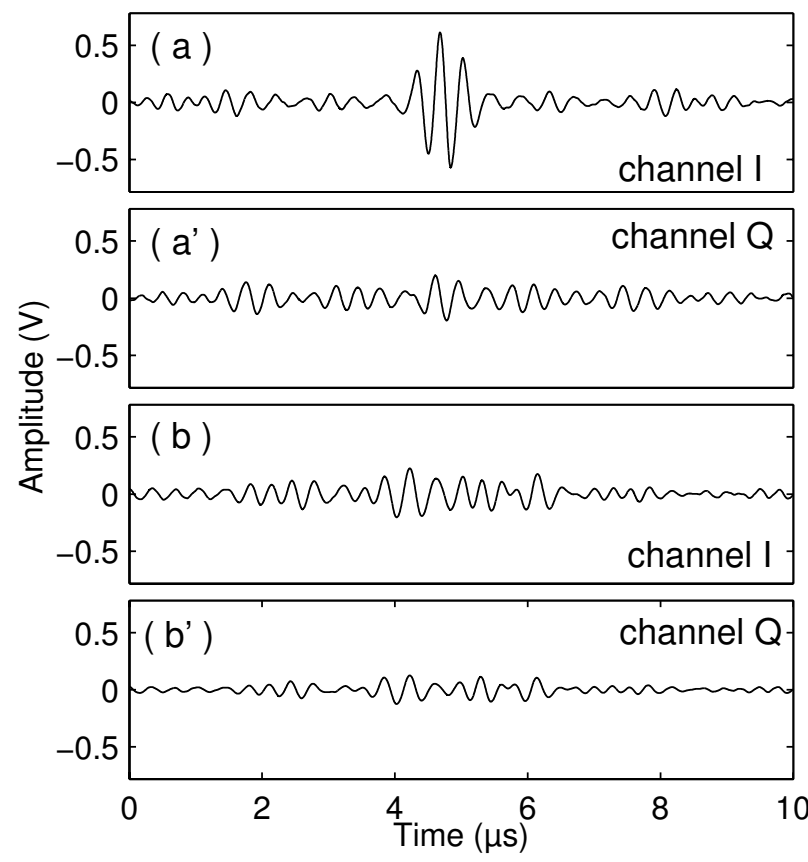

Figure 3. (a) and (a') : baseband representation of the signal received by antenna A after time-reversal. (b) and (b'): baseband representation of the signal received several wavelengths away from antenna A after timereversal.

Actually, since the reverberated wavefield has been captured by a single antenna, the time-reversal operation is not perfect. The waveform that is recreated is not the exact replica of the initial pulse: there are sidelobes around the peak on channel I, and a signal is measured on channel Q (Fig. 3. (a') ) although nothing was sent on that channel. As mentioned in the introduction, the peak-to-noise ratio in a onechannel time-reversal experiment varies as $\sqrt{\Delta \nu / \delta \nu}$. Therefore one could expect an even stronger pulse compression if the bandwidth was larger, or the correlation frequency smaller. Given the dimensions of the cavity, the Heisenberg time (i.e. the inverse of 
the mean distance between 2 successive eigenmodes of the cavity) is $t_{H} \approx 80 \mu \mathrm{s}$. But the characteristic absorption time is $t_{a}=3.6 \mu \mathrm{s}$ because of the attenuation due to the skin effect on the walls, therefore the modes are not resolved and the correlation frequency $\delta \nu$ is determined by $t_{a}$ rather than $t_{H}$. Taking $\Delta \nu=2 M H z$ and $\delta \nu=1 / t_{a} \approx 280 \mathrm{kHz}$ leads to a predicted peak-to-noise ratio roughly equal to 3 , comparable to our experimental results.

A high $\mathrm{Q}$ cavity with such a large reverberation time seems to be very far from a realistic environment. However, it should be noted that the relevant parameter is not the absolute value of the reverberation time, but rather the "spread factor", i.e. the ratio of the reverberation time to the pulse duration (or equivalently, the ratio between the bandwidth and the correlation frequency). Here we used a very reverberant chamber, but commercial transceivers with a relatively small bandwidth. The emergence of new UWB devices used in realistic indoor environment will lead to situations in which the reverberation time is not as large as in our cavity, but the bandwidth being much larger, the spreading factor will be comparable or even higher. Thus the concepts we have developed here will be transposable to realistic situations.

\subsection{Spatial Focusing}

The experiment shows that time reversal is able to compensate for multiple reverberation and recreate a short electromagnetic pulse at the source. But there is more to it: we have also verified that the amplitude of the re-created signal is stronger at antenna $A$ than anywhere else in the cavity ( Fig. 3. (b) and (b') ), i.e. the time reversed wave is spatially focused. Here once again the ratio between the peak and the spatial sidelobes is governed by the ratio $\sqrt{\Delta \nu / \delta \nu}$ [Derode et al, 2001]. Nevertheless, the actual shape of a focal spot when achieving a time reversal experiment with electromagnetic waves is still under study.

The extension of the same TR process to multiple antenna is possible. In a MISO configuration, using $\mathrm{M}$ antennas would enhance the peak-to-noise ratio by a factor $\sqrt{M}$ and further reduce the ISI. However, MISO or MIMO UWB communication experiments in reverberant media waves are difficult to implement. In the next section, we present a MISO communication experiment with ultrasonic waves propagating in a small-scale model mimicking indoor propagation of an UWB electromagnetic pulse.

\section{MISO UWB Time Reversal Communica in a Downscaled Indoor Environment Using Ultrasound}

In this section, we study time reversal communications in the context of ultrasound in a downscaled indoor environment. Our aim is to underline some features of MISO and MIMO time-reversal communication. Binary Phase Shift Keying is used to transmit digital messages and the Binary Error Rate (BER) is measured as a function of both data rate and external noise.

\subsection{Experimental Setup}

All experiments were conducted with ultrasound in a water tank. In order to mimic multipath fading and delay spread in indoor environments we have built a centimetric scale model represented in Fig. 5 . The four walls of each room are made of $3-\mathrm{mm}$ thick Plexiglass cubes $\left(100 \times 100 \times 100 \mathrm{~mm}^{3}\right)$. The corridors are $40-m m$ large. Decimetric electromagnetic wavelengths are replaced by millimetric ultrasonic ones. Needle-like piezo-electric transducers "simulate" electromagnetic antennas. The central frequency of the transducers is about $1.1 \mathrm{MHz}$ with a $30 \%$ bandwidth, which would correspond to an Ultra Wide Band electro-magnetic system with a carrier frequency of about $2 \mathrm{GHz}$ and a bandwidth of 700 $\mathrm{MHz}$. A base station made of 8 antennas is placed on the right side of the model. 8 other antennas that represent 8 possible positions for the user are randomly distributed within the model (Fig. 5). An experimental impulse response is obtained by sending a $3 \mu s$-long pulse by one of the base antennas and

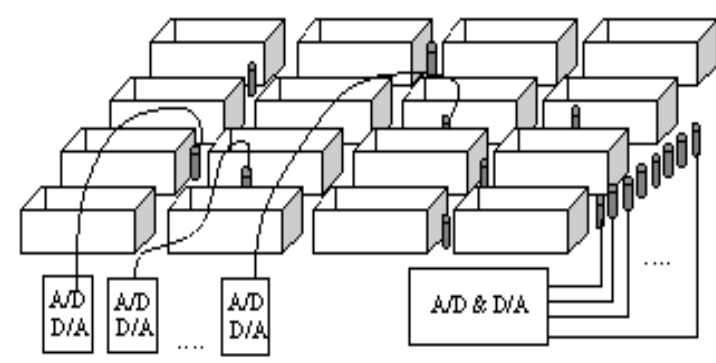

Figure 5. Small-scale model of an indoor environment. On the right side is placed a base made of 8 ultra wideband antennas. 8 other antennas, representing the possible user's positions, are randomly distributed inside the model. Each antenna is driven by its own transmit/receive electronics. 
recording the resulting wave-field at one of the receiving antennas. Thus, 64 impulse responses are recorded, corresponding to each base antenna/user antenna pair. The sampling rate is $32 M H z$. A typical impulse response is plotted in Fig. 4. In this setup we have evaluated the spread time (i.e. the rms delay spread): $\tau_{r m s} \approx 315 \mu s$.

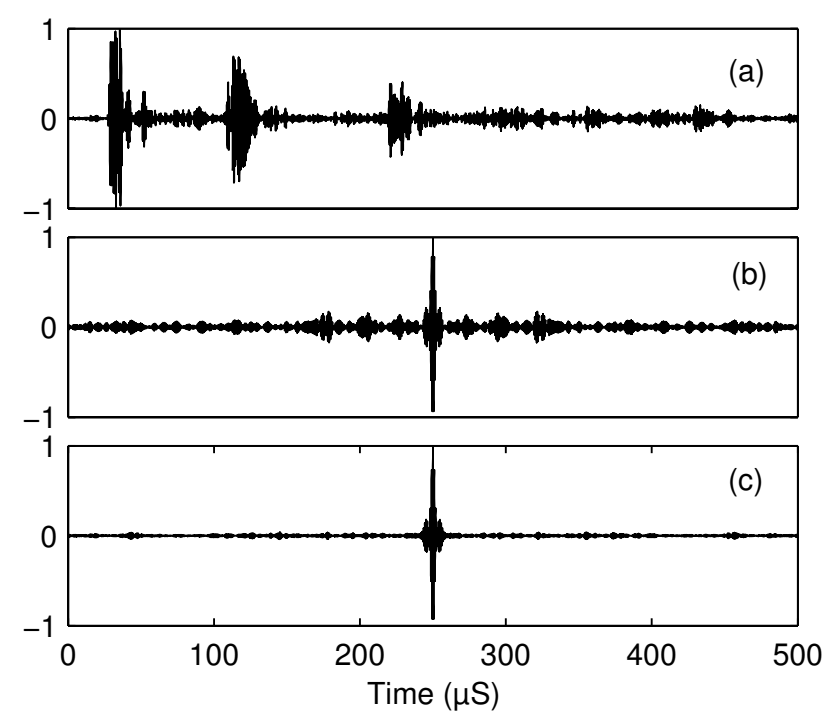

Figure 4. (a) Typical impulse response between an antenna of the base and 1 receiver. (b) and (c) compressed pulse using time reversal with respectively a 1-antenna and a 8-antenna base.

Depending on how the user is placed relatively to the base antenna, the received energy may vary significantly: a line-of-sight path gives rise to a huge and short response whereas multipathing decreases the signal amplitude. Therefore, the set of 64 impulse responses is normalized, i.e., each impulse response $h(t)$ is divided by its total energy $\int h^{2}(t) d t$. Timecompressions after TR are presented in Figs. 4.b and c. As expected the peak-to-noise ratio increases when the number of active base station antennas is increased. Nevertheless, some secondary lobes still remain because the TR is imperfect. Indeed a perfect time reversal with no secondary lobes would require a lossless medium on the one hand, and a base made of a very large amount of antennas [Fink, 1997] completely surrounding the medium on the other hand. Here, these two conditions are not fulfilled. These secondary lobes result in Inter Symbol Inteference (ISI) which is an intrinsic limitation of the imperfect TR approach.

In the following, we use TR to transmit pseudorandom sequences of bits and study the Bit Error Rate (BER). Due to the limitations of our electronics, we cannot actually transmit thousands of bits within a reasonable time frame. Therefore, instead of actually performing the communication, we compute $\mathcal{R}_{T R}$ from the set of measured impulse responses using Eq. (1), and infer the BER as follows. A 1000bit pseudo-random sequence $s(t)$ is generated. This sequence is coded using Binary Phase Shift Keying (BPSK) to form the signal $s(t)$. The received message is numerically computed convolving $s(t)$ with the signal $\mathcal{R}_{T R}$ given by Eq. (1), then demodulated and decoded by minimization of the quadratic distance on a constellation diagram. This operation is repeated until the number of wrong bits is large enough for the BER to be statistically significant. In addition when the number $\mathrm{N}$ of transmitting an-

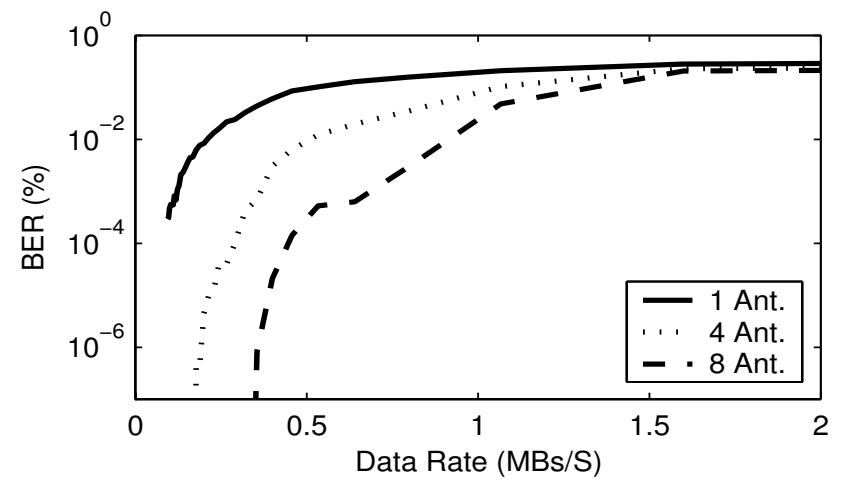

Figure 6. BER vs Data Rate for 1, 4 and 8 antennas.

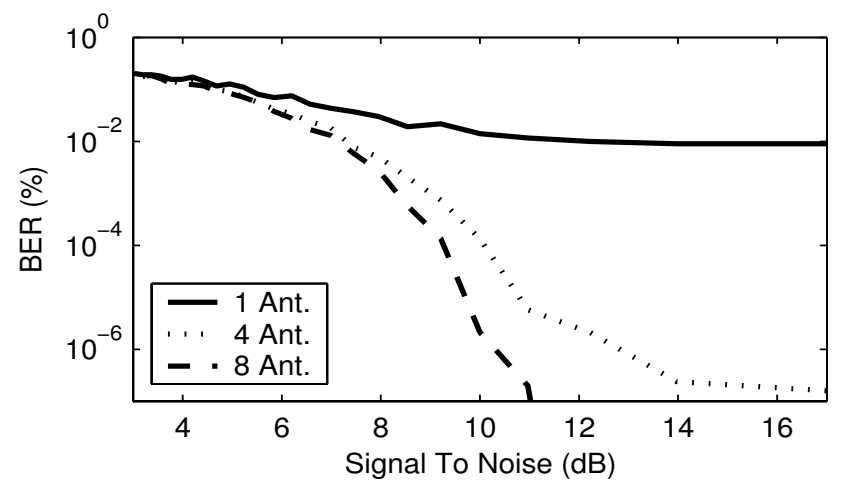

Figure 7. BER vs SNR with TR for 1,4 and 8 antennas. 
tennas is less than 8, the BER is averaged upon all possible combinations of $\mathrm{N}$ antennas picked at random among the 8 antennas of the base. Finally the $\mathrm{BER}$ is also averaged on the 8 BERs corresponding to the 8 possible positions of the receiver.

\subsection{Results}

First, we study the BER versus the data rate. Then, we investigate the robustness of the TR communication as external noise is added. In these experiments the total emitted energy remains constant regardless of the number of antennas in the base.

\subsubsection{BER versus data rate}

The measurements of the BER versus the data rate are simply performed by changing the time lag $\delta t$ between two consecutive symbols in the message $s(t)$. Each time reversed impulse response is convolved by $s(t)$. Due to the linearity and time translation invariance of the propagation medium, the symbols received by the user are separated by the same time lag $\delta t$. Obviously the data rate equals the inverse of $\delta t$. The BER is estimated for time lag ranging from $0.5 \mu \mathrm{s}(2 \mathrm{MBits} / \mathrm{s})$ to $10 \mu \mathrm{s}(100 \mathrm{kBits} / \mathrm{s})$. As shown in Fig. 6 increasing the number of antennas drastically improves the BER. Indeed as the number of base antennas is increased, the symbols' amplitude grows faster than the sidelobe level for data rates less than $1 \mathrm{MBits} / \mathrm{s}$.

\subsubsection{BER vs. external noise}

We also study the corruption of the transmission due to the presence of external noise. The SNR is defined as the ratio of the symbol amplitude to the standard deviation of the external noise; it will be expressed in dB. We assume a stationary Additive White Gaussian (AWG) external noise. The computation works as previously, but here we keep the data rate at $188 \mathrm{kbits} / \mathrm{s}$. For a base station made of $M$ antennas, the standard deviation of the AWGN is expressed as a fraction of the symbol amplitude obtained with the same number of antennas but without external noise. Fig. 7 displays the experimental results for a 1-, 4-, and 8-antenna base station. When the number of antennas is increased, the BER decreases drastically as soon as the SNR is higher than $6 \mathrm{~dB}$. Indeed for small SNR, the external noise dominates the BER, whereas the inter-symbol interference is the main contribution to the BER when the SNR is high. Increasing the number of base antennas significantly improves the BER only if we op-

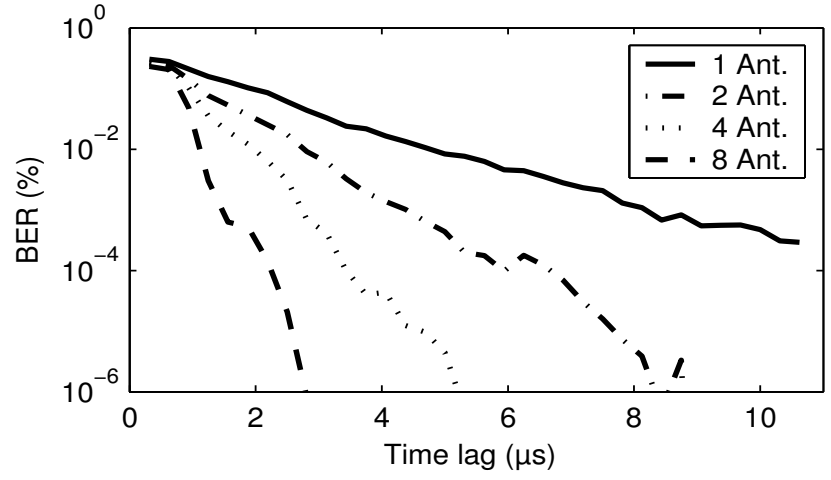

Figure 8. BER vs the time lap $\delta t$ with TR for $1,2,4$ and 8 antennas.

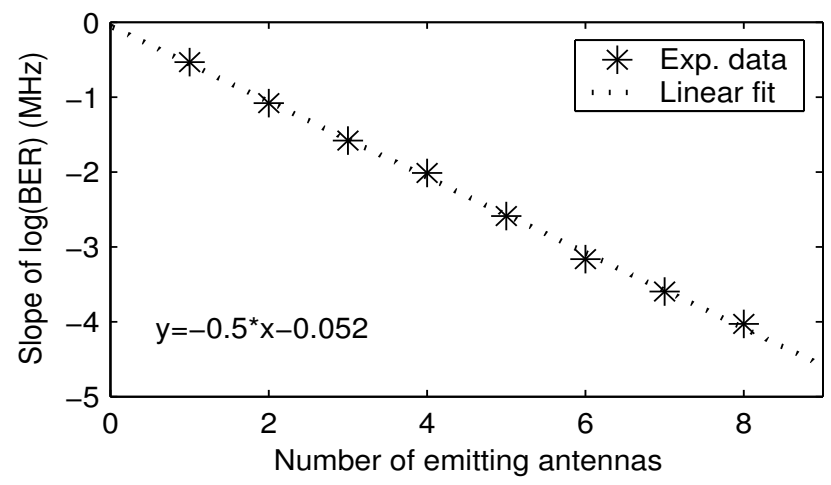

Figure 9. Slope of $\log (B E R)$ as a function of the number of antennas.

erate in an environment with an external noise lower than the "internal noise" (sidelobes) induced by the inter-symbol interferences.

\subsection{Discussion}

The logarithm of the BER seems to vary fairly linearly with the time lag $\delta t$ between the bits, i.e. the inverse of the data rate (see Fig. 8). The slope has the dimensions of a frequency, and it also varies linearly with the number of antennas, $M$ (see Fig. 9). Hence, the logarithm of the BER decreases linearly with both $M$ and $\delta t$. These experimental results are confirmed by a simple theoretical approach. In the following, we assume that the statistical fluctuations of the BER are weak enough for the BER to be considered as a good estimate of the probability of error $P_{e}$. In the case of BPSK, it is well-known that the 
probability of error is given by:

$P_{e}=Q\left(\sqrt{\frac{2 E_{b}}{N_{0}}}\right)$

where $E_{b}$ is the squared amplitude of one symbol (i.e. the energy per bit), $N_{0} / 2$ is the noise power and $Q$ is the complementary error function ${ }^{1}$. The noise power $N_{0} / 2$ is simply the addition of a "deterministic" contribution due to the inter-symbol interference (sidelobes) and external noise. If $2 E_{b} / N_{0}$ is larger than unity, a good approximation of $\log \left(P_{e}\right)$ is:

$\log \left(P_{e}\right) \approx-2 \frac{E_{b}}{N_{0}}$

The amplitude of a symbol increases as $M$ because it results from a coherent addition of the signals emitted by all the antennas of the base whereas the total sidelobe level between two symbols increases proportionally to $\sqrt{M}$ due to the incoherent sum of the inter-symbol sidelobes. Here it is assumed that the paths between the user and each antenna of the base are uncorrelated. In summary, $\log \left(P_{e}\right)$ is given by $-2 M E_{b}(1) / N_{0}(1)$, with $E_{b}(1) / N_{0}(1)$ the signal-tonoise ratio for a single antenna. When a short pulse propagates through a complex medium, scattering or reverberation spreads the initial pulse over a characteristic time $\tau$. If the scattered signal is timereversed through the same reciprocal medium, it can been shown [Derode et al, 2001] that the amplitude of the refocused pulse is proportional to $\tau \Delta \nu$, while the standard deviation of the sidelobes surrounding the peak is proportional to $\sqrt{\tau \Delta \nu}$ (see Fig. 10). Now when a sequence of bits is transmitted, while the amplitude of each symbol is still $\tau \Delta \nu$, each symbol is "contaminated" by the sidelobes of the neighboring

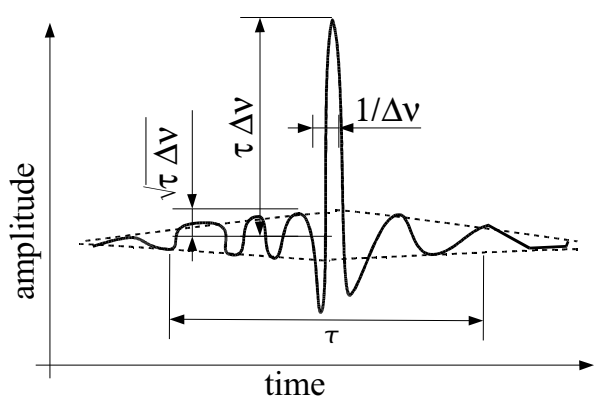

Figure 10. Schematic representation of the time compression after TR of a scattered wave issued from a single pulse. The amplitude of the pulse is proportional to $\tau \Delta \nu$. The decay time of the sidelobes is $\approx \tau$ and their typical height is $\sqrt{\tau \Delta \nu}$.

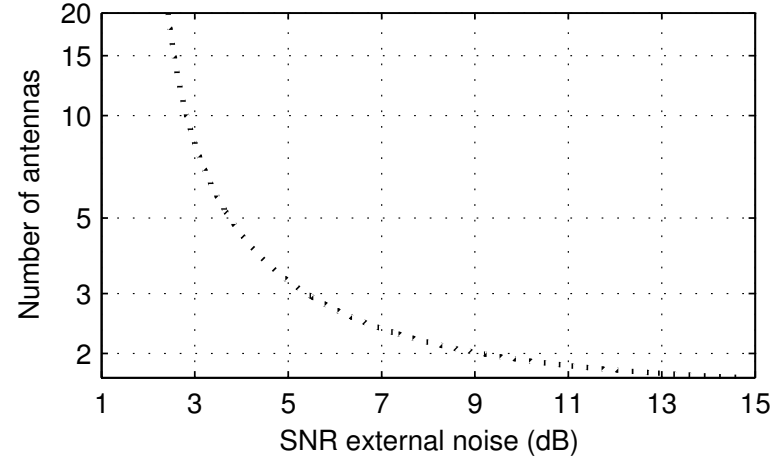

Figure 11. Number of antennas of the base versus external SNR in order to obtain a maximum transfer rate of 0.95 bit per channel use

Table 1. Maximum transfer rate D obtained without external noise for $\mathrm{M}=1$ to 4 antennas in the base

\begin{tabular}{|c||c|c|c|c|}
\hline Number of antennas & 1 & 2 & 3 & 4 \\
\hline Transfer rate & 0.8434 & 0.9762 & 0.9965 & 0.9995 \\
\hline
\end{tabular}

symbols. More precisely, the ISI results from the incoherent sum of the sidelobes due to roughly $\tau / \delta t$ symbols, so we have $N_{0}(1) \propto \tau^{2} \Delta \nu / \delta t$. Finally we have

$\frac{E_{b}(1)}{N_{0}(1)}=\Delta \nu \delta t$

Using the approximate expression for the probability of error, we finally obtain:

$\log \left(P_{e}\right) \approx-2 \Delta \nu \delta t M$

The experimental slope deduced from Fig. 9 is in fairly good agreement with Eq. 5: the value of $0.25 \mathrm{MHz}$ deduced from the linear fit corresponds roughly to the $0.35 \mathrm{MHz}$ experimental bandwidth $(\Delta \nu)$. For a Binary Symmetrical Channel (BSC), the channel capacity is given by [Proakis, 2000]:

$C=p \log _{2}(2 p)+(1-p) \log _{2}(2-2 p)$

with $p$ the transition probability $p=P(0 \mid 1)=$ $P(1 \mid 0)$. The maximum capacity of a BSC is 1 bit per channel use, it is reached when $p=0$ (no noise) or $p=1$ (every bit is surely inverted during the transmission). Stricto sensu the capacity is an ideal limit, its value should not depend on the type of modulation or on the technique of transmission; therefore it is difficult to apprehend it from an experimental point of view. However, we can use the BER as an estimated probability of error for a given technique (TR) and a given modulation (BPSK), and from Eq. 
6 estimate the maximum error-free data rate that we can hope to achieve in our experiment for the chosen modulation. Hence this transfer rate can be expressed as a function of the number $M$ of antennas, the bandwidth $\Delta \nu$ and the time lag $\delta t$ between symbols. In the following, the time lag is fixed such that $\Delta \nu \delta t=1$. When there is no external noise, the errorfree transfer rate is only limited by internal noise, i.e. the inter-symbol interference (see Tab. 1). In Fig.11 is plotted the number of antennas required to obtain a maximum transfer rate of 0.95 Bit per channel use (notice that the maximum rate is $1 \mathrm{bit}$ ) versus the signal-to-external noise ratio. When the number of antennas becomes very large, the maximum transfer rate is intrinsically limited by the sidelobes (ISI) due to the imperfections of the time-reversal. From Fig. 11 we conclude that a 0.95 -Bit transfer rate cannot be reached for less than $M=1.6$ antennas $(2$ antennas in practice!) in the base.

\section{From MISO to MIMO}

Another fundamental advantage of communications based on TR in a complex environment is that the message is spatially focused onto the receiver, with a spatial resolution that is much finer than in free space [Derode et al, 2001; Derode et al, 1995]. Besides, one can show that the typical amplitude of the spatial sidelobes also grows as $\sqrt{\tau \Delta \nu}$ while the amplitude of the refocused pulse at the source is proportional to $\tau \Delta \nu$. This is particularly interesting for MIMO communications between M transmitting and $\mathrm{K}$ receiving antennas, connected to one (MIMO-Single User) or different users (MIMOMultiple User). Whatever the configuration, because of spatial focusing it is possible to focus different bitstreams to different receivers simultaneously within the same frequency band, the degree of "overlapping" between two receivers (spatial ISI) being once again determined by $\sqrt{\tau \Delta \nu}[$ Derode et al, 2003]. In such communication schemes, the amplitude of the ISI level on each receiver will be multiplied by $\sqrt{K}$ and the error-free transfer rate can be inferred again. In that respect, the reverberating or "complex" character of the medium is essential since the number of receivers that can be simultaneously addressed depends on the number of independent paths within the medium.

\section{Conclusion}

In this paper, we demonstrated the feasibility of a one-channel TR experiment for electromagnetic waves around $2.45 \mathrm{GHz}$. This first experiment opens interesting perspectives for SISO communications in reverberant media since TR enables us to compensate for reverberation and scattering. The use of the cavity enabled us to use commercial transceivers with a low bandwidth and nevertheless to obtain a significative pulse compression (the smaller the bandwidth, the smaller the spread factor for a given environment). The emergence of new UWB devices will lead to realistic indoor situations where though there are not as many reverberations as in our cavity, the spreading factor ratio $\Delta \nu / \delta \nu$ will be at least as high as in our experiment. Thus the concepts we have developed will be transposable. TR MISO and MIMO communications have also been studied on the basis of small-scale experiments with ultrasound. In particular in a MISO communication, we have shown that TR reduces the level of intersymbol interference by a factor of $\sqrt{M}$ compared to a SISO communication, $\mathrm{M}$ being the number of antennas on the base station. This is intrinsically related to the fact that in a TR communication scheme, the transmitter has the knowledge of the channel. When the user (and not the transmitter) has the knowledge of the channel, the capacity is not improved when using a MISO scheme instead of a SISO one. This is consistent with the predictions of information theory applied to MISO communications [Paulraj, 2003, page 70]. Finally, a TR MIMO communication is well suited to take benefit of disorder and send independent messages to different users simultaneously. Indeed, the messages are naturally spatially focused onto the users which limits the cross-talking between them.

Acknowledgments. The authors wish to acknowledge the "Département de Recherche en Electromagnétisme, Laboratoire Signaux et Systèmes, Supelec", Gif-sur-Yvette, France (www.lss.supelec.fr) and particularly A. Azoulay and V. Monebhurrun for having let us use their reverberant chamber, as well as the "Conseil Régional d'Ile de France" and the "Conseil Départemental de l'Essonne" who financed it. This work is a part of the research projects developed within the "Groupement de Recherches" ImCoDe (GDR 2253, CNRS, http://lpm2c.grenoble.cnrs.fr/IMCODE/IMCODE.html).

\section{Notes}

1. $Q(x)=\left(\int_{x}^{\infty} e^{-t^{2} / 2} d t\right) / \sqrt{2 \pi}$ 


\section{References}

Blomgren, P., G. Papanicolaou, and H. Zhao (2002), Super-resolution in time-reversal acoustics, J. Acoust. Soc. Am., 111, 203-248.

Chambers, D. H. and J. G. Berryman (2004), Timereversal analysis for scatterer characterization, Phys Rev. Lett., 92, 023902.

Cheney, M. and G. Kristenson, (2001), Optimal Electromagnetic Measurments, J. Electromagnetic Waves and Apllications, 1323-1336.

Derode, A., A. Tourin and M. Fink (2001), Random Multiple Scattering of Sound, II. Is Time Reversal a self averaging process, Phys. Rev. E, 64, 036606.

Derode, A., A. Tourin, J. de Rosny, M. Tanter, S. Yon, and M. Fink (2003), Taking advantage of multiple scattering to communicate with time reversal antennas, Phys. Rev. Lett., 90, 014301-1-014301-4.

Derode, A., P. Roux, and M. Fink, Robust acoustic time reversal with high-order multiple scattering, Phys Rev. Lett., 75, 4206-4209.

Derode, A., A. Tourin, and M. Fink (1999), Ultrasonic pulse compression with one-bit time reversal through multiple scattering, J. of Appl. Phys., 85, 6343-6352.

Draeger, C. and M. Fink (1997), One-channel time reversal of elastic waves in a chaotic 2D-silicon cavity, Phys. Rev. Lett., 79, 407-410.

Edelmann, G. F., T. Akal, W.S. Hodgkiss, S. Kim, W.A. Kuperman and H.C. Song (2002), An initial demonstration of underwater acoustic communication using time reversal, IEEE J OCEANIC ENG, 3, 602-609.

Emami S.M., J. Hansen, A.D. Kim, G. Papanicolaou, A.J. Paulraj, D. Cheung, and C. Prettie, (2005), Predicted Time Reversal Performance in Wireless Communications Using Channel Measurements , IEEE Communications Letters, 9,

Fink, M. (1997), Time Reversed Acoustics, Physics Today, 50,34 .

Lerosey, G., J. de Rosny, A. Tourin, A. Derode, G. Montaldo and M. Fink (2004), Time Reversal of Electromagnetic waves, Phys. Rev. Lett., 92, 193904.

Kyritsi, P., Papanicolaou, G., Eggers, P., and A. Oprea, (2004), MISO time reversal and delay spread compression for FWA channels at $5 \mathrm{GHz}$, IEEE Antennas and Wireless Propagation Letters, 3-6, 96-99.
Oestges, C., Kim, A.D., Papanicolaou, G. and A.J. Paulraj, (2005), Characterization of space-time focusing in time reversed random fields, IEEE Trans. Antennas and Prop, 53, 283-293.

Henty, B.E., and D.D. Stancil, (2004), MultipathEnabled Super-Resolution for rf and Microwave Communication using Phase-Conjugate Arrays, Physical Review Letters, 93, 243904-1 243904-4.

Micolau, G., M. Saillard, and P. Borderies (2003), DORT method as applied to ultrawideband signals for detection of buried objects, IEEE Trans. Geosci. Remote Sens., 41, 1813-1820.

Montaldo, G., G. Lerosey, A. Derode, A. Tourin, J. de Rosny and M. Fink (2004), Telecommunication in a disordered environment with iterative time reversal, Waves Random Media, 14, 287-302.

Moustakas, A.L., H.U. Baranger, L. Balents, A.M. Sengupta and S.H. Simon (2000), Communication through a diffusive medium: Coherence and capacity, Science, 287-5451, 287-290.

Nguyen, H.T., J.B. Andersen, G.F. Pedersen, (2005), The potential use of time reversal technique in multiple elements antenna systems, IEEE Communications Letters, 9

Paulraj, A., R. Nabar and D. Gore (2003), Introduction Space-Time Wireless Communications, Cambridge university Press.

Paulraj, A. J., D. A. Gore, R. U. Nabar, and H. Bölcskei, (2004), An overview of MIMO communications - A key to Gigabit wireless, Proc. of the IEEE, 92-2, 198-218.

Pepper, D. M. (1982), Non-linear optical-phase conjugation, Opt. Eng., 21, 156-183.

Proakis, J.G. (2000), Digital Communications, McGrawHill Higher Education.

G. Lerosey, Laboratoire Ondes et Acoustique, ESPCI, Université Paris VII, UMR 7587, 10 rue Vauquelin, 75005 Paris, France. (geoffroy.lerosey@loa.espci.fr)

(Received 